\title{
Analysis of high speed machining center spindle dynamic unit structure performance \\ Yuan guowei
}

Liaoning jidian polytechnic,Dan dong china

yyuanguowei@163.com

Keywords:spindle; dynamic performance; finite element

\begin{abstract}
As the core components of high-speed machining center, Electric spindle realizes "zero transmission machine", the main content of this paper is the response and stability analysis of critical speed, unbalance of spindle unit, and the transient excitation system in a variety of response and the torsional natural frequency and response.
\end{abstract}

\section{Introduction}

Characteristic finite element method is generally used to calculate spindle unit, especially the stiffness characteristics, high calculation precision. But the analysis of the spindle unit, finite element software is not easy and bearing analysis theory combining, need to be calculated through intergral generalized load should be relative and bearing load, and the bearing load and deformation relation is linearized.

With the requirements of the dynamic performance of the spindle to improve, various factors influencing the dynamic characteristics of spindle should be comprehensive consideration of the dynamic characteristics of spindle in analysis at, to make the results more close to the actual situation.

\section{Calculation and analysis of characteristics of spindle bearing}

As the key technology of high speed spindle bearing in, directly determine the load capacity, working performance of spindle(high speed,high precision, high rigidity movement), the working life and the spindle dynamic, static performance. Ball bearing dynamic performance is better than the traditional ceramic ball bearings, therefore, in the high speed machining center, ceramic ball bearing electric spindle is usually adopted. Improvement of spindle bearing lubrication on spindle speed plays an important role, the general use of oil mist lubrication or oil injection lubrication.

1)Analysis of axial force angle contact ball

Product nominal diameter bearing $D_{m}$ and spindle speed $n(\mathrm{r} / \mathrm{min})$ is called the rated speed coefficient, When the rated speed $D_{m}{ }^{n}$ coefficient reaches $3.0 \times 10^{6} \mathrm{~mm} / \mathrm{min}$ above, Electric spindle basically are installed in high speed rolling bearing. Angular contact ball bearings for, When using the constant preload, the centripetal force or thermal expansion radial offset will cause the inner ring radial size broadening or rolling body, resulting axial offset bearing center. When using rigid bearing arrangement, the causes of bearing inner load increase. Because the bearing pressure angle change caused by the stiffness reduction will cause the spindle dynamic stiffness reduction, then will reduce non flutter condition of material removal rate[1].

The bearing stiffness $K_{B}$ by the rolling body size, rolling groove curvature and pressure angle decision. But the dynamics performance and pre bearing load, cutting force, speed and heat deformation related. Bearing shear stiffness in one case only useful: the dynamic performance of the spindle is nonlinearsystem.

For angular contact ball bearing, and can bear radial load $F_{r}$ and axial load $F_{a}$. Pressure line stress ball force after the intersect at a point on the axis, the so-called pressure angle, As shown in Figure 1. 


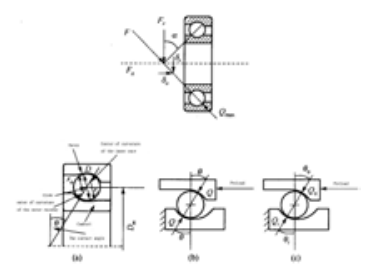

Figure 1 Force analysis of angular contact ball bearing

At the same time under radial and axial loads, Bearing inner ring and the outer ring are respectively produce relative displacement in the radial and axial $\delta_{r}$ amd $\delta_{\alpha}$, In this case, each ball bearing load is not consistent. each ball bearing deformation can be calculated by the following formula:

$$
\begin{aligned}
& \delta_{\psi}=\delta_{\alpha} \sin \alpha+\delta_{r} \cos \alpha \cos \psi \\
& \delta_{\psi}=0
\end{aligned}
$$

In the formula, $\alpha$ is the bearing contact angle; $\psi$ Is the angle between the center and the maximum load of each ball bearing rolling body center.

the ball load

$$
Q_{\psi}=1.149 \times 10^{11}\left(\mathrm{D}_{b} \cdot \delta_{\psi}^{3}\right)^{1 / 2}
$$

the ball force balance

$$
\begin{aligned}
& F_{r}=\sum Q_{\psi} \cos \alpha \sin \psi \\
& F_{\alpha}=\sum Q_{\psi} \sin \alpha
\end{aligned}
$$

2) Bearing axial radial static stiffness estimation

For approximate calculation of bearing stiffness, first assume that the bearing under load contact angle remains unchanged, and regardless of bearing raceway diameter and the influence on the main curvature and function, the derivation of the angular contact bearing stiffness and deformationof simplified calculation formula. Bearings only bear axial force, the channel of the bearing force, therefore we can obtain axial strain $\delta_{\alpha}$ and axial stiffness $K_{\alpha}$

$$
\begin{aligned}
& \delta_{\alpha}=4.45 \times 10^{-4} F_{\alpha}^{1 / 3} Z^{2 / 3} D^{-1 / 3}\left(\sin \alpha_{0}\right)^{-5 / 3} \\
& K_{\alpha}=3.37079 \times 10^{3} F_{\alpha}^{1 / 3} Z^{2 / 3} D^{1 / 3}\left(\sin \alpha_{0}\right)^{5 / 3}
\end{aligned}
$$

the radial stiffness $k_{r}$, assuming that only the radial deformation of bearing $\delta_{r}$, and the axial deformation $\delta_{r}$ is zero, at this time the bearings only bear radial force, you can obtain the radial deformation $\delta_{r}$ and radial stiffness $k_{r}$

$$
\begin{aligned}
& \delta_{r}=1.1895 \times 10^{-4} F_{r}^{2 / 3} Z^{2 / 3} D^{-1 / 3}\left(\sin \alpha_{0}\right)^{-5 / 3} \\
& K_{r}=1.26096 \times 10^{3} F_{r}^{1 / 3} Z^{2 / 3} D^{1 / 3}\left(\sin \alpha_{0}\right)^{5 / 3}
\end{aligned}
$$

3) Stiffness calculation of bearing axial preload of angular contact

Bearing proper pre tight can increase in stiffness and the life of shaft, in the supporting structure of spindle, preload mainly fixed pressure pretension and positioning pretension in two forms, the latter is applied more widely[3].

In the practical application, Suppose bearing radial force, the inner, outer only radial displacement without axialdisplacement. If the bearing preload is $F_{\alpha 0}$, approximate that each ball deformation consistent, Each ball load is $Q=F_{\alpha 0} /\left(Z_{0} \sin \alpha\right)$, Among them $Z_{0}$ is the number of balls, for

$$
\delta=4.23 \times 10^{-8}\left(\mathrm{Z}^{2} \sin ^{2} \alpha \cdot \mathrm{D}_{b}\right)^{-1 / 3} \cdot F_{\alpha 0}^{2 / 3}
$$

axial bearing relative displacement is

$\delta_{\alpha 0}=\delta / \sin \alpha=4.23 \times 10^{-8}\left(\mathrm{Z}^{2} \sin ^{5} \alpha \cdot \mathrm{D}_{b}\right)^{-1 / 3} \cdot F_{\alpha 0}^{2 / 3}$

axial pre tightening after, as to subject to radial load, the inner and outer ring relative radial displacement, assuming the bearing preload for axial positioning pretension, and then subjected to 
radial loading, axial displacement axial displacement of pre tight, Such is the elastic deformation of the ball at

$\delta_{\psi=\delta_{\alpha 0} \sin \alpha+\delta_{r} \cos \alpha \cos \psi}$

load of each ball is

$Q_{\psi}=1.149 \times 10^{11} \cdot D_{b}^{1 / 2}\left(\delta_{\alpha 0} \sin \alpha+\delta_{r} \cos \alpha \cos \psi\right)^{3 / 2}$

according to stress analysis, radial load $F_{r}$ is equal to the algebraic each ball load and radial component,

$F_{r}=\sum Q_{\psi} \cos \alpha \cos \psi=1.149 \times 10^{11} \cdot D_{b}^{1 / 2}\left(\delta_{\alpha 0} \sin \alpha+\delta_{r} \cos \alpha \cos \psi\right)^{3 / 2} \cos \alpha \cos \psi$

As can be seen from the above equation, With $\delta_{r}$ increases, $F_{r}$ Increases, but the situation varies increased preload changes. the formula for $\delta_{r}$ the derivative, you can get the radial stiffness

$K_{r}=1.149 \times 10^{11} \cdot D_{b}^{1 / 2} \cdot \cos ^{2} \alpha \cdot \frac{3}{2} \cdot\left(\delta_{\alpha 0} \sin \alpha\right)^{1 / 2} \cdot \sum \cos ^{2} \psi$

For ceramic ball bearings, preload available under the action of its radial stiffness is

$K_{r}=1.3 \times 17.73 \times \cdot D_{b}^{1 / 2} \cdot\left(Z^{2} \cdot D_{b}\right)^{1 / 3} \cdot \frac{\cos ^{2} \alpha}{\sin ^{1 / 3} \alpha}\left(F_{\alpha 0}\right)^{1 / 3}(\mathrm{~N} / \mu \mathrm{m})$

as can be seen from the above analysis, the relationship between the bearing load and deformation non-linear relationship. bearing stiffness is a function of the load, load, the greater stiffness. This is the calculation of the ceramic ball bearing preload must account for the cause. In actual calculation, always want to have a stiffness value for reference. When the external load can not be determined, Computing often taken as a dynamic load bearing $1 / 10$ rated load bearing.

\section{Spindle cell structure analysis of the inherent characteristics of computing}

Establishing an electric spindle and bearing support finite element model, bearing with spring damper units, dynamic characteristics of a motor spindle machining center model calculation model shown in Figure 2, figure three, four as the main component of the second and fourth-order mode shapes.

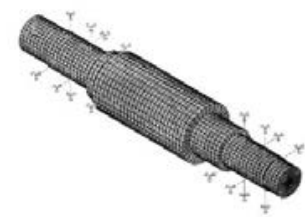

Figure 2 spindle dynamic characteristic calculation model

Vibrational structure can be expressed as each order natural vibration mode of the linear combination. Where low-level natural modes of vibration of the structure of the higher order effects of large, the more low-level the greater the impact, low order modes of the dynamic characteristics of the structure play a decisive role[4]. So the use of Subspace modal extraction method to calculate the spindle first five natural frequencies and mode shapes as shown in Table I .

Table I. Table Natural Frequencies And Mode Shapes Of A Spindle

\begin{tabular}{|l|l|l|}
\hline Order & Frequency $/ \mathrm{HZ}$ & Modes \\
\hline 1 & 0 & Reverse \\
\hline 2 & 1170.5 & A first bending \\
\hline 3 & 1170.5 & A first bending \\
\hline 4 & 2129.9 & Second-order bending \\
\hline 5 & 2129.9 & Second-order bending \\
\hline
\end{tabular}

One can see from the table, wo, three natural frequencies are equal, Modes were first bending and first swing, So its performance is orthogonal modes; Similarly, four, five bands as well. Figure III and IV, respectively, a first bending axis and second bend, As can be seen made by Ansys spindle modal analysis, get a good space simulation results. 
To ensure the machining accuracy and security, When the electric spindle work can not exceed $75 \%$ of the maximum speed of one of the first critical speed. As can be seen from Table 1, This article first critical speed electric spindle is $60 \times 1170.5=70230 \mathrm{r} / \mathrm{min}$, the maximum operating speed is $30000 \mathrm{r} / \mathrm{min}$, far less than the first-order critical speed, So the electric spindle design is reasonable, Can effectively avoid the resonance region. To ensure the precision of the spindle[5].

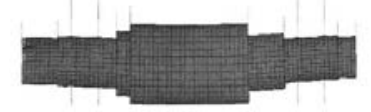

Figure 3 second-order modes

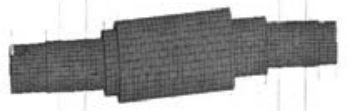

Figure 4, fourth-order modes

\section{4 the impact on the pre-load and speed spindle unit performance}

Generally, All modes of natural frequencies increase with increased preload, because the bearing stiffness increases will follow; Simultaneously with the increase of spindle speed decreases, Because the centrifugal force will be increased. To more clearly compare the simulation and measurement results, Figure 5 gives a fixed spindle speed or pre-load, second-order effects on the natural frequency of the pre-load and the spindle speed. When the pre-load is increased from $600 \mathrm{~N}$ to $1800 \mathrm{~N}$ when a large, Natural frequency increases from $1068 \mathrm{~Hz}$ to $1142 \mathrm{~Hz}$. Correspondingly, When the spindle speed is increased by a stationary, The natural frequency is reduced from $1140 \mathrm{~Hz}$ to $1090 \mathrm{~Hz}$. Experiments and analysis show that, Pre-load and speed on the dynamic characteristics of electric spindle unit has a certain impact.
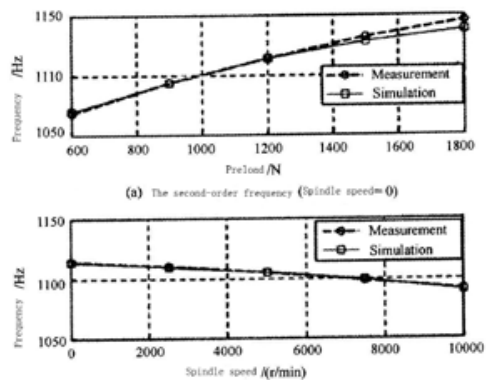

Figure 5 preload affects spindle speed and the natural frequency of the second order

\section{Conclusions}

Through the analysis of high-speed machining center electric spindle structure dynamic performance, spindle cells improved dynamic performance measures:(1) select the appropriate precision bearings and related parts of precision, spindle rotation accuracy can be improved.(2) Choose the right spindle structure size, choose a good bearing stiffness, power transmission and reasonable layout position and orientation can be good to improve the rigidity spindle unit.(3) Minimize the length of the front end portion of the bearing structure of the spindle, to maximize the stiffness and damping of the front bearing, the front bearing to maximize the accuracy of the spindle unit can be improved vibration resistance.

\section{References}

[1] "High-speed electric spindle CNC machine control technology" Science Press, Wu yuhou, Zhang Lixiu editor.

[2] "CNC machine tool spindle cell electric technology" Yu-thick editor Machinery Industr y Press. 
[3] "High-speed machining center performance modeling and optimization" Science Press editor Wang Shuting

[4] "CNC Machine Tool Spindle cell technology" Machinery Industry Press Wu yuhou e ditor.

[5] " Key technology research power permanent magnet synchronous motor spindle drive" Beijing University Press Yujiabin editor. 